\title{
Early determinants for the development of undernutrition in an older general population: Longitudinal Aging Study Amsterdam
}

\author{
Janneke Schilp ${ }^{1 *}$, Hanneke A. H. Wijnhoven ${ }^{1}$, Dorly J. H. Deeg ${ }^{2}$ and Marjolein Visser ${ }^{1,2}$ \\ ${ }^{1}$ Department of Health Sciences and the EMGO Institute for Health and Care Research, Faculty of Earth and Life Sciences, \\ VU University Amsterdam, De Boelelaan 1085, 1081 HV Amsterdam, The Netherlands \\ ${ }^{2}$ Department of Epidemiology and Biostatistics, VU University Medical Centre, De Boelelaan 1118, 1081 HV Amsterdam, \\ The Netherlands
}

(Received 12 July 2010 - Revised 25 January 2011 - Accepted 25 January 2011 - First published online 30 March 2011)

\begin{abstract}
Undernutrition may be an important modifiable risk factor for poor clinical outcomes in older individuals. To achieve earlier detection or prevention of undernutrition, more information is needed about risk factors for the development of undernutrition in community-dwelling older individuals. The objective was to identify early determinants of incident undernutrition in a prospective population-based study. Baseline data (1992-3) on socio-economic, psychological, medical, functional, lifestyle and social factors of 1120 participants aged 65-85 years of the Longitudinal Aging Study Amsterdam were used. Undernutrition, defined as a BMI $<20 \mathrm{~kg} / \mathrm{m}^{2}$ or self-reported involuntary weight loss $\geq 5 \%$ in the last 6 months, was assessed every 3 years during a 9-year follow-up period. Cox proportional-hazards regression analysis was used to investigate the association between early determinants at baseline and incident undernutrition. In 9 years, 156 participants (13.9\%) developed undernutrition. In univariate analyses, female sex, depressive symptoms, anxiety symptoms, multiple chronic diseases, high medication use (women), poor appetite, no alcohol use $v$. light alcohol use, loneliness, not having a partner, limitations in performing normal activities due to a health problem, low physical performance (participants aged $<75$ years) and reporting difficulties walking stairs (participants aged $<75$ years) were statistically significantly associated with incident undernutrition. In a multivariate model, poor appetite and reporting difficulties walking stairs (participants aged $<75$ years) remained early determinants. The results of the present study can be used to identify subgroups of older individuals with increased risk of undernutrition and to identify modifiable determinants for the purpose of prevention of undernutrition.
\end{abstract}

Key words: Undernutrition: Early determinants: Older individuals

Undernutrition can be defined as a disorder of nutritional status resulting from reduced nutrient intake or impaired metabolism $^{(1)}$. There is increasing awareness that undernutrition may be an important modifiable ${ }^{(2)}$ risk factor for poor clinical outcomes in older individuals in developed countries $^{(3-10)}$. Associations are found with a reduced functional status ${ }^{(11,12)}$, physical impairment ${ }^{(13)}$, reduced quality of life $^{(3,10)}$, hospitalisation and mortality ${ }^{(4,6,7)}$.

In community-based populations prevalence rates range, depending on the study population and the used definition of undernutrition, from $2 \%$ diagnosed by a low albumin level $(<350 \mathrm{mg} /)^{(14)}$, to $24 \%$ diagnosed by the Nutritional Screening Initiative ${ }^{(15)}$. Previous research mainly focused on examining the effects of treating undernutrition ${ }^{(2)}$. Surprisingly, little to no attention is paid to prevention of undernutrition in community-dwelling older individuals. However, before prevention programmes can be developed, information is needed on determinants for undernutrition so that high-risk groups and modifiable determinants can be identified.

Previous studies have identified several determinants for undernutrition in older individuals, such as older age ${ }^{(16,17)}$, depression $^{(17-19)}$, poor cognitive functioning ${ }^{(18)}$, impaired physical functioning ${ }^{(5,9,18)}$, difficulties with biting and chewing $^{(9,18)}$, dementia ${ }^{(9)}$, co-morbidity ${ }^{(5,16,20,21)}$, poor appetite $^{(18,21)}$, vision problems ${ }^{(21)}$ and stress ${ }^{(21)}$. However, many of these studies were performed in institutionalised older individuals ${ }^{(9,18,19)}$. Most importantly, most of these studies had a cross-sectional design ${ }^{(5,9,16,18-21)}$, whereby causality of an association cannot be established. Only one study among community-dwelling older individuals had a prospective design ${ }^{(17)}$. However, the size of the study population was relatively small ( $n$ 579) and a limited number of determinants was examined in this study. For example, medication use, number of chronic diseases, appetite, alcohol use, 
education level and physical performance were not examined as determinants. Therefore, more longitudinal studies are needed in the community, regarding an extensive multidisciplinary set of determinants. The aim of the present prospective study was to identify early determinants of incident undernutrition in a large population-based sample of men and women aged $65-85$ years.

\section{Subjects and methods}

\section{Subjects}

Data were collected in the context of the Longitudinal Aging Study Amsterdam (LASA), an ongoing cohort study focusing on physical, emotional, cognitive and social functioning in an older population. A random sample of older individuals aged 55-85 years, stratified by age, sex, level of urbanisation and expected 5-year mortality, was drawn from the population registers of eleven municipalities in areas in the west (Amsterdam and vicinity), northeast (Zwolle and vicinity) and south (Oss and vicinity) of the Netherlands. In total, 3107 participants were enrolled in the main baseline examination, conducted between September 1992 and September 1993. Follow-up examinations were performed every 3 years. At each examination, a general interview in the individual's home was followed after 4-6 weeks by a medical interview during which medical tests were performed. Participants also completed a self-administered questionnaire. Further information about the sampling and data-collection procedures have been described elsewhere in detail ${ }^{(22)}$.

After exclusion of participants aged $<65$ years, the present study included 2141 participants aged $\geq 65$ years who participated in the main baseline examination. Participants with missing data on BMI and/or self-reported weight change at baseline ( $n$ 454) and participants with missing data on BMI and/or self-reported weight change at any follow-up examination because they died ( $n$ 343) or dropped out of the study ( $n$ 100) were excluded. Participants who were undernourished at baseline ( $n$ 124) were excluded to be able to investigate incident undernutrition (see the section on Undernutrition below for the applied definition of undernutrition). The final study sample therefore consisted of 1120 participants: 543 men and 577 women.

The present study was conducted according to the guidelines laid down in the Declaration of Helsinki and all procedures involving human subjects were approved by the Medical Ethics Committee of the VU University Medical Centre (Amsterdam, The Netherlands). Written informed consent was obtained from all participants.

\section{Undernutrition}

Body height and weight were measured during the medical interview in a standing position wearing light indoor clothing without shoes. A wall-mounted stadiometer was used to measure height to the nearest $\mathrm{mm}$. If no accurate measurement of height could be obtained $(n 71 ; 6.3 \%)$ due to the recorded particularities 'not able to stand', 'shoes', 'kyphosis', 'scoliosis', or 'unknown reasons', height was imputed by either: (1) a valid follow-up measurement of height ( $n$ 55); or (2) a sex-specific prediction rule based on age and knee height ( $n$ 15) $)^{(23)}$; or (3) self-reported height ( $n$ 1). Knee height of the left leg was measured with a Mediform sliding caliper (Medical Express, Beaverton, OR, USA) with the knee and ankle joints fixed at $90^{\circ}$ angles. Height at baseline was used in the calculation of BMI at follow-up examinations, because height may decrease over time due to spinal deformities. Body weight was measured to the nearest $0 \cdot 1 \mathrm{~kg}$ using a standard balance beam scale. In deviating situations adjustments were made for clothing $(-1 \mathrm{~kg})$, corset $(-1 \mathrm{~kg})$ and shoes $(-1 \mathrm{~kg})^{(24)}$. In all medical interviews a self-reported weight was obtained, which was used when no measured weight was available (seven, six, five and five participants, respectively, at baseline, and at 3-, 6- and 9-year followups). BMI was calculated as body weight ( $\mathrm{kg}$ ) divided by height $(\mathrm{m})$ squared. Weight change in the last 6 months was assessed by asking the following question: 'Did your weight change in the last 6 months?'. Furthermore, the amount of weight change $(\mathrm{kg})$ and the reason for weight change were asked. Involuntary weight loss was defined as weight loss due to disease, loss of appetite, (psycho)social factors or 'unknown reasons'. Undernutrition was defined as either a BMI $<20 \mathrm{~kg} / \mathrm{m}^{2}$ or self-reported involuntary weight loss $\geq 5 \%$ in the last 6 months ${ }^{(25)}$. This definition was applied to determine both undernutrition at baseline (these participants were excluded) as well as incident undernutrition.

\section{Determinants of undernutrition}

All information on determinants was obtained at baseline during the general interview ( $n$ 1120), except for medication use which was assessed during the medical interview ( $n$ 1113) and self-reported pain and problems with biting and chewing which were assessed in the self-administered questionnaire ( $n$ 936).

Socio-economic factors. Education level was categorised into high (university, college and higher vocational education), medium (general secondary, intermediate vocational, general intermediate and lower vocational education) and low education (elementary education or elementary education not completed). Monthly household income was categorised into tertiles: high ( $\geq 1035$ euro), medium (625-1035 euro), low $(<625$ euro) and missing. If the participant had a partner living in the household, income was multiplied by $0 \cdot 7^{(26)}$.

Psychological factors. Cognitive functioning was measured with the Mini-Mental State Examination, with scores ranging from 0 to $30^{(27)}$, whereby scores $\leq 23$ were defined as a poor cognitive status ${ }^{(28)}$. Depression was measured with the Dutch translation of the Center for Epidemiologic Studies Depression scale, with scores ranging from 0 to $60^{(29)}$. Scores $\geq 16$ were defined as depression ${ }^{(30)}$. Anxiety was measured with the anxiety subscale of the Hospital Anxiety and Depression Scale, with scores ranging from 0 to $21^{(31)}$. The cut-off point $\geq 7$ was used to determine anxiety disorders $^{(32)}$. 
Medical factors. The presence of chronic diseases was determined by explicitly asking the participants whether they had any of the following diseases: cardiac diseases (including myocardial infarction), peripheral atherosclerosis, stroke, diabetes mellitus, obstructive lung disease (asthma, chronic bronchitis or pulmonary emphysema), arthritis (rheumatoid arthritis or osteoarthritis) or cancer. The accuracy of self-report data for these diseases as compared with general practitioners' information was shown to be adequate ${ }^{(33)}$. To define co-morbidity three categories were created: no chronic disease; one chronic disease; two or more chronic diseases. Medication use was determined by having the interviewer check the containers of drugs that the respondent was taking, with or without prescription, and three categories were created: no medication use; use of one or two medications; and use of three or more medications. Appetite during the last week was assessed with the following question from the Dutch translation of the Center for Epidemiologic Studies Depression Scale ${ }^{(29)}$ : 'I did not feel like eating; my appetite was poor', with answering categories: (1) 'rarely or none of the time'; (2) 'some or little of the time'; (3) 'occasionally or moderate amount of the time'; and (4) 'most or all of the time'. For appetite two categories were created: no problems with appetite (answer 1); and poor appetite last week (answers 2-4). Subjective pain was determined asking five questions from a subscale of the Nottingham Health Profile ${ }^{(34)}$. Sum scores were calculated and divided into no pain (score 5), pain (score 6-10), and missing. Problems with biting and chewing were assessed by asking 'Are you able to bite or chew hard food?'. Participants answering 'almost never' or 'some of the time' were categorised as having no problem, and those answering 'often' or 'most of the time' as having problems. A third category for missing values was made.

Functional factors. Visual impairment, with glasses or contact lenses if needed, was ascertained by two items: (1) read the fine print in a newspaper and (2) recognise a face at a $4 \mathrm{~m}$ distance. Hearing impairment, with a hearing aid if needed, was ascertained by two items: (1) follow a conversation with one individual and (2) follow a conversation in a group of four individuals. For visual and hearing impairment two categories were created: 'none' and 'one or two items with some difficulty'. Limitation of normal activities due to a health problem was assessed by asking 'Are health problems limiting your normal daily activity?'. Participants answering 'yes, severely' and 'yes, mild' were categorised as having limitations and those answering 'no' as not having limitations. Physical performance was measured with three standardised performance tests: chair stands, tandem stand and walk test, each with scores ranging from 0 to 4 . Scores on the three tests were summed, resulting in a total score ranging from 0 (poor performance) to $12^{(35)}$. Difficulty walking stairs was assessed by the question 'Can you climb up and down a staircase of 15 steps without stopping?' with answering categories (1) 'yes'; (2) 'yes, with difficulty'; (3) 'not able without help'; and (4) 'cannot'. For difficulty walking stairs two categories were created: no difficulties (answer 1) and difficulties (answers 2-4) ${ }^{(36)}$.
Lifestyle factors. Smoking status was categorised into: current smoker; former smoker; never smoker; and missing. Former smokers who stopped smoking more than 15 years ago were classified as never smoker ${ }^{(37)}$. Alcohol use was based on the number of days per month drinking alcohol and the number of alcohol consumptions each time. Four categories were created: no alcohol; light; moderate; and (very) excessive alcohol use ${ }^{(38)}$. Physical activity in the previous 2 weeks was assessed using the validated Longitudinal Aging Study Amsterdam (LASA) Physical Activity Questionnaire (LAPAQ) ${ }^{(39)}$, whereby information on the frequency and duration of walking, bicycling, household activities, and sport activities was obtained. Total physical activity was expressed in min per $\mathrm{d}$.

Social factors. Loneliness was measured with a Dutch validated loneliness scale ${ }^{(40)}$. The scale consisted of eleven items, with three possible answers: (0) 'no'; (1) 'more or less'; and (2) 'yes'. We used a cut-off score of $\geq 3$, as applied by others ${ }^{(41)}$, to identify participants suffering from loneliness. Individuals without a partner inside or outside the household were defined as not having a partner. Type of housing was observed at the main interview, whereby a distinction was made between independent living, including those who receive home care, and not independent living, including institutionalised participants.

\section{Statistical analyses}

At 3, 6 and 9 years' follow-up, the incidence of undernutrition was determined according to the definition described earlier. Baseline characteristics of the group developing undernutrition and the group not developing undernutrition were compared using the $\chi^{2}$ test for dichotomous and categorical variables and the independent-samples $t$ test for continuous variables. Time to event was defined as the number of days between the baseline examination and the first follow-up examination where undernutrition was diagnosed. Followup time of censored participants was calculated using the date of the 9-year follow-up examination, the date of the last follow-up examination or the date of death, whichever came first.

Cox proportional-hazards regression analysis was used to investigate the association between potential determinants of undernutrition at baseline and the incidence of undernutrition during a 9-year follow-up period. To investigate the proportional hazard assumption, $\log (-\log ($ survival $))$ curves of categorical determinants were visually inspected and for continuous variables a time interaction test was performed, with statistical significance based on a $P$ value $<0 \cdot 01$. Effect modification by age and sex was examined by adding interaction terms to the univariate regression model. In the case of a statistically significant interaction $(P<0.05)$, associations were presented stratified by age $(<75$ years and $\geq 75$ years) and/or sex. The linearity of the association between each continuous covariate and undernutrition was checked by adding a quadratic term to the model.

All determinants that were found to be statistically significantly associated with the development of undernutrition in 
Table 1. Baseline characteristics of the study sample

(Mean values and standard deviations or percentages)

\begin{tabular}{|c|c|c|c|c|c|c|c|}
\hline & \multicolumn{2}{|c|}{$\begin{array}{c}\text { All } \\
(n 1120)\end{array}$} & \multicolumn{2}{|c|}{$\begin{array}{l}\text { Undernutrition } \\
\qquad(n 156)^{\star}\end{array}$} & \multicolumn{2}{|c|}{$\begin{array}{c}\text { No undernutrition } \\
(n \text { 964) } \dagger\end{array}$} & \multirow[b]{2}{*}{$P$} \\
\hline & Mean & SD & Mean & SD & Mean & SD & \\
\hline \multicolumn{8}{|l|}{ Demographic factors } \\
\hline Female $(\%)$ & \multicolumn{2}{|c|}{51.5} & \multicolumn{2}{|c|}{$60 \cdot 3$} & \multicolumn{2}{|c|}{$50 \cdot 1$} & 0.02 \\
\hline Age (years) & $74 \cdot 1$ & $5 \cdot 7$ & $74 \cdot 2$ & $6 \cdot 0$ & $74 \cdot 1$ & $5 \cdot 7$ & 0.77 \\
\hline \multicolumn{8}{|l|}{ Socio-economic factors (\%) } \\
\hline \multicolumn{8}{|l|}{ Education } \\
\hline Low & \multicolumn{2}{|c|}{$43 \cdot 2$} & \multicolumn{2}{|c|}{47.4} & \multicolumn{2}{|c|}{$42 \cdot 5$} & 0.46 \\
\hline Medium & \multicolumn{2}{|c|}{$45 \cdot 5$} & \multirow{2}{*}{\multicolumn{2}{|c|}{$\begin{array}{l}41.0 \\
11.5\end{array}$}} & \multicolumn{2}{|c|}{$46 \cdot 3$} & \\
\hline High & & & & & & & \\
\hline Income in euro & & & & & & & \\
\hline Low & & & & & & & 0.98 \\
\hline Medium & & & & & & & \\
\hline High & & & & & & & \\
\hline Missing & & & & & & & \\
\hline Psychological factors (\%) & & & & & & & \\
\hline Poor cognitive status (MMSE $\leq 23$ ) & & & & & & & 0.48 \\
\hline Depressive symptoms (CES-D $\geq 16$ ) & & & & & & & $<0.01$ \\
\hline Anxiety symptoms (HADS $\geq 7)$ & & & & & & & 0.03 \\
\hline Medical factors (\%) & & & & & & & \\
\hline Number of chronic diseases & & & & & & & \\
\hline No chronic disease & & & & & & & $<0.01$ \\
\hline One chronic disease & & & & & & & \\
\hline Two or more chronic diseases & & & & & & & \\
\hline Medication use & & & & & & & \\
\hline No medication & & & & & & & $<0.01$ \\
\hline One or two medications & & & & & & & \\
\hline Three or more medications & & & & & & & \\
\hline Poor appetite & & & & & & & $<0.01$ \\
\hline Pain & & & & & & & \\
\hline No pain & & & & & & & 0.48 \\
\hline Pain & & & & & & & \\
\hline Missing & & & & & & & \\
\hline Problems with biting and chewing & & & & & & & \\
\hline Never or some of the time & & & & & & & 0.63 \\
\hline Often or most of the time & & & & & & & \\
\hline Missing & & & & & & & \\
\hline Functional factors & & & & & & & \\
\hline Vision problems (\%) & & & & & & & 0.84 \\
\hline Hearing problems (\%) & & & & & & & 0.47 \\
\hline $\begin{array}{l}\text { Limitation of normal activities due } \\
\text { to a health problem }(\%)\end{array}$ & & & & & & & $<0.01$ \\
\hline Physical performance test $(0-12)$ & $7 \cdot 0$ & $2 \cdot 6$ & $6 \cdot 7$ & $2 \cdot 5$ & $7 \cdot 0$ & $2 \cdot 6$ & $0 \cdot 10$ \\
\hline Difficulties walking stairs (\%) & & & & & & & $<0.01$ \\
\hline Lifestyle factors & & & & & & & \\
\hline $\mathrm{BMI}\left(\mathrm{kg} / \mathrm{m}^{2}\right)$ & $27 \cdot 2$ & 3.9 & $26 \cdot 4$ & 4.5 & $27 \cdot 3$ & $3 \cdot 8$ & $<0.01$ \\
\hline Smoking (\%) & & & & & & & \\
\hline Never & & & & & & & 0.58 \\
\hline Former & & & & & & & \\
\hline Current & & & & & & & \\
\hline Alcohol use (\%) & & & & & & & \\
\hline No alcohol use & & & & & & & 0.13 \\
\hline Light & & & & & & & \\
\hline Moderate & & & & & & & \\
\hline (Very) excessive & & & & & & & \\
\hline Physical activity (min/d) & 161.5 & $192 \cdot 6$ & $146 \cdot 3$ & $117 \cdot 0$ & 164.0 & $202 \cdot 1$ & 0.12 \\
\hline Social factors (\%) & & & & & & & \\
\hline Loneliness (score $\geq 3$ ) & & & & & & & 0.06 \\
\hline Having a partner & & & & & & & 0.01 \\
\hline Living independently & & & & & & & $0 \cdot 16$ \\
\hline
\end{tabular}

MMSE, Mini-Mental State Examination; CES-D, Center for Epidemiologic Studies Depression scale; HADS, Hospital Anxiety and Depression Scale.

${ }^{*}$ Participants developing undernutrition during the 9-year follow-up.

$\dagger$ Participants not developing undernutrition during the 9 -year follow-up. 
Table 2. Associations of socio-economic, lifestyle, social, psychological, medical and functional factors at baseline and 9-year incident undernutrition

(Hazard ratios (HR) and 95\% confidence intervals)

\begin{tabular}{|c|c|c|c|c|c|}
\hline & \multirow[b]{2}{*}{$n \ddagger$} & \multicolumn{2}{|c|}{ Univariate model } & \multicolumn{2}{|c|}{ Multivariate model } \\
\hline & & $\mathrm{HR}$ & $95 \% \mathrm{Cl}$ & $\mathrm{HR}$ & $95 \% \mathrm{Cl}$ \\
\hline Female & 1120 & 1.40 & $1.01,1.92$ & 0.73 & $0.38,1.39$ \\
\hline \multicolumn{6}{|l|}{ Age } \\
\hline$<75$ years & 625 & 1 & Reference & 1 & Reference \\
\hline$\geq 75$ years & 495 & 1.30 & $0.95,1.79$ & 0.88 & $0.29,2.63$ \\
\hline \multicolumn{6}{|l|}{ Education } \\
\hline Low & 484 & 1 & Reference & - & \\
\hline Medium & 510 & 0.78 & $0.56,1.09$ & - & \\
\hline High & 126 & 0.94 & $0.56,1.58$ & - & \\
\hline \multicolumn{6}{|l|}{ Income (euro) } \\
\hline Low & 307 & 1 & Reference & & \\
\hline Medium & 418 & 0.98 & $0.66,1.44$ & & \\
\hline High & 265 & 0.89 & $0.57,1.39$ & & \\
\hline Missing & 130 & 0.93 & $0.54,1.62$ & & \\
\hline Poor cognitive status & 1116 & 0.94 & $0.49,1.78$ & & \\
\hline Depressive symptoms (yes $v$. no) & 1112 & 1.96 & $1.32,2.93$ & 0.89 & $0.52,1.52$ \\
\hline Anxiety symptoms (yes v. no) & 1094 & 1.75 & $1 \cdot 11,2 \cdot 78$ & 1.26 & $0.72,2 \cdot 21$ \\
\hline \multicolumn{6}{|l|}{ Number of chronic diseases } \\
\hline No chronic disease & 252 & 1 & Reference & 1 & Reference \\
\hline One chronic disease & 422 & 1.23 & $0.76,2.00$ & $1 \cdot 10$ & $0.64,1.88$ \\
\hline Two or more chronic diseases & 442 & 2.08 & $1.31,3.28$ & 1.32 & $0.75,2.33$ \\
\hline \multicolumn{6}{|l|}{ Medication use } \\
\hline No medication & 354 & 1 & Reference & 1 & Reference \\
\hline One or two medications, male & 195 & $0.47^{*}$ & $0.23,0.95$ & $1 \cdot 10^{*}$ & $0.60,2.02$ \\
\hline One or two medications, female & 209 & $0.36^{*}$ & $0.76,2.41$ & $0.39^{*}$ & $0.18,0.83$ \\
\hline Three or more medications, male & 154 & 1.51 & $0.86,2.66$ & 1.80 & $0.99,3.27$ \\
\hline Three or more medications, female & 201 & 2.57 & $1.50,4.38$ & 1.03 & $0.54,1.96$ \\
\hline Poor appetite & 1119 & 1.99 & $1.32,3.00$ & 1.63 & $1.02,2.61$ \\
\hline \multicolumn{6}{|l|}{ Pain } \\
\hline No pain & 585 & 1 & Reference & & \\
\hline Pain, male & 100 & 1.29 & $0.70,2.37$ & & \\
\hline Pain, female & 152 & 1.37 & $0.82,2 \cdot 27$ & & \\
\hline Missing, male & 109 & $0.62^{*}$ & $0.29,1.33$ & & \\
\hline Missing, female & 174 & $1.62^{*}$ & $1.01,2.61$ & & \\
\hline \multicolumn{6}{|l|}{ Problems with biting and chewing } \\
\hline Never or some of the time & 304 & 1 & Reference & - & \\
\hline Often or most of the time & 596 & 1.81 & $0.57,1.16$ & - & \\
\hline Missing & 220 & 0.83 & $0.52,1.32$ & - & \\
\hline Vision problems (yes v. no) & 1096 & 1.00 & $0.65,1.52$ & - & \\
\hline Hearing problems (yes $v$. no) & 1095 & 1.42 & $0.93,2 \cdot 16$ & - & \\
\hline Limitation of normal activities due to a health problem & 1118 & 1.76 & $1.28,2.43$ & $1 \cdot 20$ & $0.81,1.77$ \\
\hline Physical performance test score, aged $<75$ years & 601 & $0.89 \dagger$ & $0.81,0.96$ & 0.98 & $0.89,1.08$ \\
\hline Physical performance test score, aged $\geq 75$ years & 475 & $1 \cdot 01 \dagger$ & $0.92,1.11$ & 1.06 & $0.95,1.18$ \\
\hline \multicolumn{6}{|l|}{ Difficulties walking stairs } \\
\hline No difficulties walking stairs & 824 & 1 & Reference & 1 & Reference \\
\hline Difficulties walking stairs, aged $<75$ years & 115 & $2.50 \dagger$ & $1.59,3.91$ & $1.91 \dagger$ & $1.14,3.22$ \\
\hline Difficulties walking stairs, aged $\geq 75$ years & 177 & $1.08 \dagger$ & $0.67,1.75$ & $0.88 \dagger$ & $0.51,1.50$ \\
\hline \multicolumn{6}{|l|}{ Smoking } \\
\hline Never & 720 & 1 & Reference & - & \\
\hline Former & 167 & 0.82 & $0.50,1.33$ & - & \\
\hline Current & 232 & 1.08 & $0.73,1.61$ & - & \\
\hline \multicolumn{6}{|l|}{ Alcohol use } \\
\hline No alcohol use & 249 & 1 & Reference & 1 & Reference \\
\hline Light & 598 & 0.67 & $0.46,0.98$ & 0.82 & $0.55,1.96$ \\
\hline Moderate & 228 & 0.82 & $0.52,1.30$ & $1 \cdot 11$ & $0.67,1.83$ \\
\hline (Very) excessive & 38 & $1 \cdot 16$ & $0.52,2.58$ & 1.42 & $0.58,3.46$ \\
\hline Physical activity & 1120 & 0.99 & $0.997,1.000$ & & \\
\hline Loneliness (yes v. no) & 1105 & 1.47 & $1.06,2.04$ & $1 \cdot 11$ & $0.75,1.64$ \\
\hline Having a partner (no $v$. yes) & 1118 & 1.70 & $1 \cdot 24,2 \cdot 33$ & 1.37 & $0.92,2.02$ \\
\hline Living independently (no $v$. yes) & 1120 & $3 \cdot 13$ & $0.44,22 \cdot 33$ & & \\
\hline
\end{tabular}

* Statistically significant interaction with sex $(P<0.05)$.

† Statistically significant interaction with age $(P<0.05)$.

$\ddagger$ Number of participants for the categories of the determinants in which the univariate analyses were performed. 
the univariate analyses were included in a multivariate model. Multicollinearity diagnostics (with linear regression analysis) were used to identify possible linear dependencies among determinants. In the case of a variance inflation factor value above $10^{(42)}$, one of the involved determinants was removed from the multivariate analysis. In the case of a statistical significant interaction $(P<0.05)$ between a covariate and age and/or sex in the univariate model, interaction terms were added for these covariates in the multivariate model as well. Results were presented as hazard ratios with 95\% CI. Two-sided $P$ values of 0.05 were considered statistically significant. Analyses were performed using SPSS software (version 15.0; SPSS, Inc., Chicago, IL, USA).

\section{Results}

The baseline characteristics of the study sample are presented in Table 1. During the 9-year follow-up period (mean follow-up 7.1 (SD 2.2) years) 156 out of 1120 participants $(13.9 \%)$ developed undernutrition. Of these, 114 participants (73.1\%) met the criterion $\geq 5 \%$ self-reported involuntary weight loss in the last 6 months during any of the follow-up examinations', thirty participants (19.2\%) met the criterion 'BMI $<20 \mathrm{~kg} / \mathrm{m}^{2}$ during any of the follow-up examinations' and twelve participants $(7.7 \%)$ met both criteria. Of the participants developing undernutrition, fifty-three died before the end of the 9-year follow-up. At the 3- and 6-year followups the cumulative incidences of undernutrition were 6.5 and $11 \cdot 4 \%$, respectively. A total of 281 participants $(25 \cdot 1 \%)$ died during the follow-up without a prior classification of undernutrition.

The univariate and multivariate associations between potential determinants and incident undernutrition are shown in Table 2. For all analyses, the proportional hazard assumption was valid. The following sociodemographic and lifestyle factors were found to be associated $(P<0.05)$ with the risk of developing undernutrition in the univariate analyses: female sex; no alcohol use $v$. light alcohol use; loneliness and not having a partner. The following psychological, medical and functional factors were identified $(P<0.05)$ : depressive symptoms; anxiety symptoms; presence of two or more chronic diseases $v$. no chronic diseases; poor appetite; experiencing limitations in performing normal activities due to a health problem; use of three or more medications (women only) $v$. no medication; low physical performance test score (age $<75$ years only) and reporting difficulties walking stairs (age $<75$ years only) $v$. reporting no difficulties. Using one or two medications $v$. no medication use was associated with a reduced risk of developing undernutrition in men.

Because no multicollinearity was found, all statistically significant determinants of the univariate analyses were included in a multivariate model. Interaction terms were tested for medication use $\times$ sex, physical performance test score $\times$ age, and reporting difficulties walking stairs $\times$ age. The interaction terms of sex $x$ using one or two medications (and not using three or more medications) and age $\times$ reporting difficulties walking stairs were statistically significant in the multivariate model $(P<0 \cdot 05)$. Poor appetite and reporting difficulties walking stairs were the only determinants that statistically significantly increased the risk of developing undernutrition in the multivariate model. Thereby, reporting difficulties walking stairs increased the risk only in participants aged $<75$ years. Using one or two medications statistically significantly decreased the risk compared with using no medication in women. In men no statistical significance was found for medication use.

An additional analysis was performed to investigate whether poor appetite could be seen as an intermediate risk factor for undernutrition. For example, depression, chronic disease or medication use might lead to undernutrition (partly) through a poor appetite. We therefore repeated the multivariate model, excluding the variable poor appetite. The results are presented in Table 3 and show that even after exclusion of poor appetite these factors are not associated with incident undernutrition in the multivariate model.

Fig. 1 shows the cumulative incidence of undernutrition according to those with a normal appetite and those with a poor appetite. Fig. 2 shows the cumulative incidence of undernutrition for those who reported no difficulties walking stairs and those who did report difficulties walking stairs, stratified for age.

\section{Discussion}

To our knowledge, this is the first prospective study to investigate early determinants of incident undernutrition from a large multidisciplinary set of variables in community-dwelling older individuals. The 9-year incidence of undernutrition in this population-based sample of men and women aged 65 to 85 years was $13.9 \%$. Our multi-disciplinary approach showed that several determinants were statistically significantly associated with an increased risk of developing undernutrition in univariate analyses, including: female sex, depressive symptoms, anxiety symptoms, having two or

Table 3. Associations of depressive symptoms, chronic disease and medication use at baseline and 9-year incident undernutrition (multivariate analysis $\dagger$ )

(Hazard ratios (HR) and $95 \%$ confidence intervals)

\begin{tabular}{lll}
\hline & $\mathrm{HR}$ & $95 \% \mathrm{Cl}$ \\
\hline $\begin{array}{lll}\text { Depressive symptoms (yes } v . \text { no) } \\
\text { Number of chronic diseases }\end{array}$ & 0.99 & $0.58,0.66$ \\
$\quad$ No chronic disease & 1 & Reference \\
$\quad$ One chronic disease & 1.11 & $0.65,0.91$ \\
$\quad$ Two or more chronic diseases & 1.30 & $0.74,0.29$ \\
Medication use & & \\
$\quad$ No medication & 1 & Reference \\
$\quad$ One or two medications, male & $0.39^{*}$ & $0.18,0.83$ \\
$\quad$ One or two medications, female & $1.08^{*}$ & $0.59,0.98$ \\
$\quad$ Three or more medications, male & 1.05 & $0.55,0.99$ \\
$\quad$ Three or more medications, female & 1.75 & $0.96,0.20$ \\
\hline
\end{tabular}

* Statistically significant interaction with $\operatorname{sex}(P<0.05)$.

$\dagger$ Adjusted for all statistically significant determinants of the univariate model, except appetite (sex, depressive symptoms, anxiety symptoms, number of chronic diseases, medication use, loneliness, not having a partner, limitations in performing normal activities due to a health problem, low physical performance test score and reporting difficulties walking stairs). 


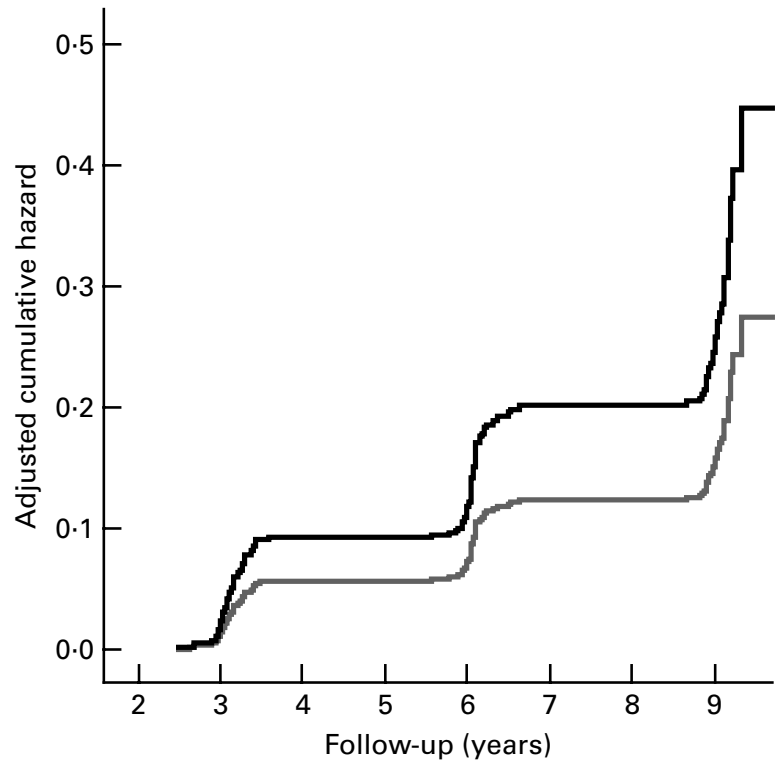

Fig. 1. Cumulative hazard of incident undernutrition according to appetite at baseline (normal appetite, - (gray line); poor appetite, - (black line)), adjusted for the variables included in the multivariate model (see Table 2).

more chronic diseases, using three or more medications (women only), poor appetite, experiencing limitations in performing normal activities due to a health problem, low physical performance test score (participants aged $<75$ years only), reporting difficulties walking stairs (participants aged $<75$ years only), loneliness and not having a partner. Light alcohol use, compared with no alcohol use, statistically significantly decreased the risk of developing undernutrition. In the multivariate analysis, poor appetite and reporting difficulties walking stairs (participants aged $<75$ years) were the only remaining statistically significant determinants that increased the risk of incident undernutrition. Furthermore, light medication use decreased the risk of undernutrition in women.

The associations between most of these determinants and undernutrition have been suggested based on previous cross-sectional studies, but have not been confirmed in a prospective study among community-living older individuals. A cross-sectional relationship between poor functional status and prevalent undernutrition (as assessed by the Mini Nutritional Assessment) in older individuals was previously reported for hospital patients ${ }^{(18)}$, nursing home patients ${ }^{(9)}$ and community-dwelling individuals ${ }^{(5)}$. In the present study, function-related factors were statistically significantly associated with incident undernutrition in univariate analyses in the group aged $65-75$ years. The oldest group ( $\geq 75$ years) reported more functional limitations walking stairs compared with the youngest group (65-75 years), 36\% compared with $18 \%$. The oldest group also had a lower physical performance test score (mean 6.1 (SD 2.5)) compared with the youngest group (mean 7.7 (SD 2.5)). Although poor functional status is more prevalent at higher ages, only when they occur at an earlier age do they seem to be associated with the development of undernutrition. In the present study, the strongest early functional determinant of incident undernutrition was reporting difficulties walking stairs, independent of health status.

An earlier prospective study of Johansson et al. ${ }^{(17)}$ reported associations between older age and depressive symptoms (assessed with the Geriatric Depression Scale) and incident risk of undernutrition (as assessed by the Mini Nutritional Assessment), which were comparable with the present results in the univariate analyses. In the multivariate analyses, these associations became statistically insignificant in the present study but remained statistically significant in the study of Johansson et $a l^{(17)}$. This may be explained by the fact that we adjusted for a wider range of covariates. Furthermore, in the study of Johansson et $a l .{ }^{(17)}$ lower self-perceived health predicted incident risk of undernutrition, but this could not be replicated in the present study. This can be explained by the fact that our used definition of undernutrition does not include questions directly related to poorer health status like the Mini Nutritional Assessment does.

(a)

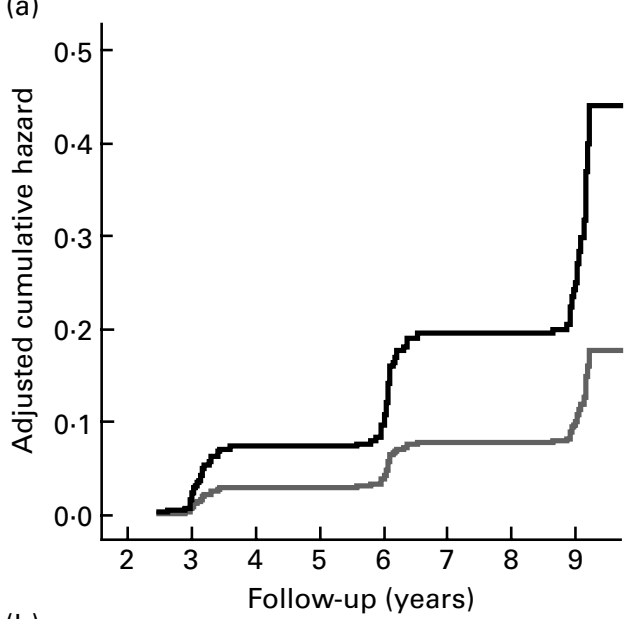

(b)

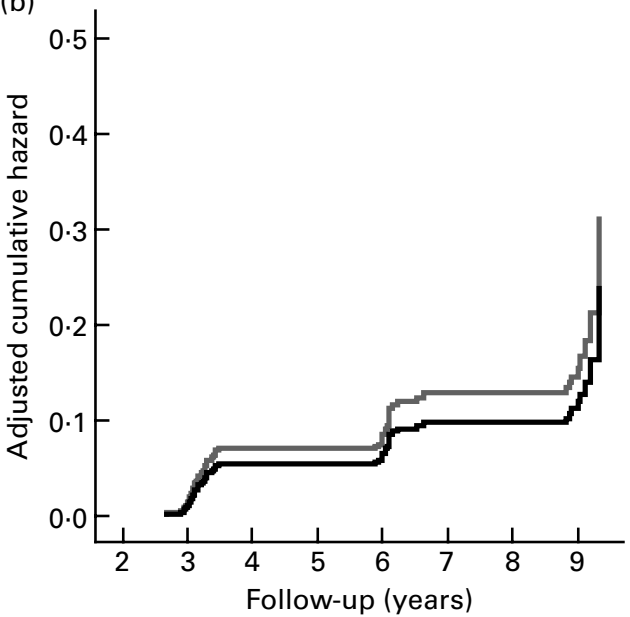

Fig. 2. Cumulative hazard of incident undernutrition according to reporting of difficulties walking stairs (no difficulties in walking stairs, - (gray line); difficulties in walking stairs, - (black line)) at baseline in those under 75 years (a) and in those aged 75 years or older (b), adjusted for the variables included in the multivariate model (see Table 2). 
Following the strong association between poor appetite and incident undernutrition in the present study, it is remarkable that only a few earlier studies have investigated this association. The cross-sectional association between appetite and prevalent undernutrition was found in hospital patients ${ }^{(18)}$ and in home-care patients where poor appetite was independently associated with lower energy and protein intakes ${ }^{(21)}$. Some determinants that were found to be associated with prevalent undernutrition in earlier cross-sectional studies, such as cognitive problems ${ }^{(18)}$, difficulties with biting and chewing $^{(9,18)}$ and vision problems ${ }^{(21)}$, were not confirmed in our prospective study. These results may suggest that these determinants are associated with present undernutrition but do not predict the development of undernutrition over time. An alternative explanation could be that the prevalence and/ or severity of these problems is lower in community-dwelling individuals and that these problems will only have an impact on nutritional status in institutionalised patients ${ }^{(18,21)}$. The present study also identified other determinants of undernutrition that have not yet been studied $^{(43)}$. For example, participants with anxiety had an increased risk of developing undernutrition.

In the present study, a decreased risk of incident undernutrition was found for light alcohol use compared with no alcohol use. Previous studies have shown that light alcohol use reduces morbidity and mortality ${ }^{(44,45)}$. The beneficial effect of light alcohol consumption on morbidity and mortality could be partly explained by the high energy content of alcoholic drinks, which may influence the risk of developing undernutrition.

Using one or two medications compared with no medication use seems to be protective for the development of undernutrition in the present study. A clarification for this apparently controversial outcome could be that single medication-users probably more often use preventive medication, which may have a beneficial effect on health. Furthermore, participants using one or two medications could be more 'healthy minded' compared with non-users.

An important strength of the present study is the unique substantial multidisciplinary set of factors that could be included to examine their association with the development of undernutrition, ranging from social factors to psychological and medical factors. Another strength of the present study is the use of a definition of undernutrition that was confirmed in recent literature to be an appropriate definition in community-dwelling older individuals ${ }^{(46-48)}$. In this definition self-reported weight change is used to investigate weight loss. A study among 4716 men aged 57-78 years showed that self-reported weight change corresponded well with changes in measured weight ${ }^{(49)}$. Furthermore, in contrast to many other studies, a distinction was made between involuntary and voluntary weight loss in the present study to determine undernutrition. This is important, because individuals with involuntary weight loss have different characteristics and health outcomes compared with individuals with voluntary weight loss ${ }^{(49)}$.

There are some limitations of the present study. First, following the design of our cohort study, we only had measurements of undernutrition at 3-year intervals. Undernutrition can develop rapidly, for example, in the case of acute disease ${ }^{(50)}$. It is possible that some participants developed undernutrition in between two examinations and were censored because they died. Participants could also be recovered from undernutrition before a follow-up measurement took place. This limitation probably resulted in an underestimation of the cumulative incidence of undernutrition in the present study. Furthermore, some potential determinants could not be investigated because they were not assessed in the study, such as difficulties with shopping or cooking, taste and smell problems or low nutritional intake, which are mentioned in earlier reviews to be associated with undernutrition $^{(43,46,50,51)}$. Future prospective studies are needed to confirm and extend our findings.

The present prospective study provides insight into the factors that could contribute to the development of undernutrition. We conclude that several socio-economic, psychological, medical, functional, lifestyle and social factors were associated with the future development of undernutrition in community-dwelling older individuals. In multivariate analyses, only a poor appetite and reporting difficulties walking stairs (aged $<75$ years) remained in the model as determinants of undernutrition. Both determinants can easily be assessed by simple questions and are therefore useful in screening and early recognition of community-dwelling older individuals at risk of undernutrition. Other determinants found in the univariate models may be underlying factors of a poor appetite and difficulties walking stairs and may therefore help to target preventive interventions.

\section{Acknowledgements}

The present study is based on data collected in the context of the Longitudinal Aging Study Amsterdam (LASA), which is largely funded by the Ministry of Health, Welfare, and Sports of the Netherlands.

Authors' contributions were as followed: J. S., H. A. H. W. and M. V. carried out the design of the study. J. S. performed the statistical analyses and drafted the manuscript. H. A. H.W., D. J. H. D. and M. V. gave significant advice concerning interpretation of the results and critical review of the manuscript. All authors reviewed and approved of the manuscript before submission.

The authors had no personal or financial conflicts of interest.

\section{References}

1. August DA \& Kushner RF (1995) The 1995 A.S.P.E.N standards for nutrition support: hospitalized patients. Nutr Clin Pract 10, 208-219.

2. Milne AC, Potter J \& Vivanti A, et al. (2009) Protein and energy supplementation in elderly people at risk from malnutrition. The Cochrane Database of Systematic Reviews 2009, issue 2, CD003288. http://www.mrw.interscience. wiley.com/cochrane/clsysrev/articles/CD003288/frame.html 
3. Amarantos E, Martinez A \& Dwyer J (2001) Nutrition and quality of life in older adults. J Gerontol A Biol Sci Med Sci 56, Spec. no. 2, 54-64.

4. Corrada MM, Kawas CH, Mozaffar F, et al. (2006) Association of body mass index and weight change with all-cause mortality in the elderly. Am J Epidemiol 163, 938-949.

5. Han Y, Li S \& Zheng Y (2009) Predictors of nutritional status among community-dwelling older adults in Wuhan, China Public Health Nutr 12, 1189-1196.

6. Knudtson MD, Klein BE, Klein R, et al. (2005) Associations with weight loss and subsequent mortality risk. Ann Epidemiol 15, 483-491.

7. Locher JL, Roth DL, Ritchie CS, et al. (2007) Body mass index, weight loss, and mortality in community-dwelling older adults. I Gerontol A Biol Sci Med Sci 62, 1389-1392.

8. Newman AB, Yanez D, Harris T, et al. (2001) Weight change in old age and its association with mortality. J Am Geriatr Soc 49, 1309-1318.

9. Suominen M, Muurinen S, Routasalo P, et al. (2005) Malnutrition and associated factors among aged residents in all nursing homes in Helsinki. Eur J Clin Nutr 59, 578-583.

10. Thomas DR (2001) The critical link between health-related quality of life and age-related changes in physical activity and nutrition. I Gerontol A Biol Sci Med Sci 56, M599-M602.

11. Cereda E, Valzolgher L \& Pedrolli C (2008) Mini nutritional assessment is a good predictor of functional status in institutionalised elderly at risk of malnutrition. Clin Nutr 27, 700-705

12. Salvi F, Giorgi R, Grilli A, et al. (2008) Mini Nutritional Assessment (short form) and functional decline in older patients admitted to an acute medical ward. Aging Clin Exp Res 20, 322-328.

13. Ferdous T, Cederholm T, Razzaque A, et al. (2009) Nutritional status and self-reported and performance-based evaluation of physical function of elderly persons in rural Bangladesh. Scand J Public Health 37, 518-524.

14. Dirren H, Decarli B, Lesourd B, et al. (1991) Nutritional status: haematology and albumin. Euronut SENECA investigators. Eur J Clin Nutr 45, Suppl. 3, 43-52.

15. Marshall JA, Lopez TK, Shetterly SM, et al. (1999) Indicators of nutritional risk in a rural elderly Hispanic and nonHispanic white population: San Luis Valley Health and Aging Study. J Am Diet Assoc 99, 315-322.

16. Margetts BM, Thompson RL, Elia M, et al. (2003) Prevalence of risk of undernutrition is associated with poor health status in older people in the UK. Eur J Clin Nutr 57, 69-74.

17. Johansson Y, Bachrach-Lindstrom M, Carstensen J, et al (2009) Malnutrition in a home-living older population: prevalence, incidence and risk factors. A prospective study. J Clin Nurs 18, 1354-1364.

18. Feldblum I, German L, Castel H, et al. (2007) Characteristics of undernourished older medical patients and the identification of predictors for undernutrition status. Nutr J 6, 37

19. Thomas DR, Zdrowski CD, Wilson MM, et al. (2002) Malnutrition in subacute care. Am J Clin Nutr 75, 308-313.

20. Martin CT, Kayser-Jones J, Stotts NA, et al. (2007) Risk for low weight in community-dwelling, older adults. Clin Nurse Spec 21, 203-211.

21. Payette H, Gray-Donald K, Cyr R, et al. (1995) Predictors of dietary intake in a functionally dependent elderly population in the community. Am J Public Health 85, 677-683.

22. Deeg DJH, van Tilburg T, Smit JH, et al. (2002) Attrition in the Longitudinal Aging Study Amsterdam. The effect of differential inclusion in side studies. J Clin Epidemiol 55 $319-328$
23. Chumlea WC, Roche AF \& Steinbaugh ML (1985) Estimating stature from knee height for persons 60 to 90 years of age. J Am Geriatr Soc 33, 116-120.

24. Frank E \& Dunlop AL (2000) What does a patient's outfit weigh? Fam Med 32, 595-596.

25. Elia M (2001) MAG screening tool and guidelines set to combat malnutrition. Guidel Pract 4, 41-50.

26. Koster A, Bosma H, Broese van Groenou MI, et al. (2006) Explanations of socioeconomic differences in changes in physical function in older adults: results from the Longitudinal Aging Study Amsterdam. BMC Public Health 6, 244.

27. Folstein MF, Folstein SE \& McHugh PR (1975) 'Mini-mental state'. A practical method for grading the cognitive state of patients for the clinician. J Psychiatr Res 12, 189-198.

28. Wind AW, Schellevis FG, van Staveren G, et al. (1997) Limitations of the Mini-Mental State Examination in diagnosing dementia in general practice. Int $J$ Geriatr Psychiatry 12, $101-108$.

29. Radloff LS (1977) The CES-D scale: a self-report depression scale for research in the general population. ApplPsych Meas 1, 385-401.

30. Berkman LF, Berkman CS, Kasl S, et al. (1986) Depressive symptoms in relation to physical health and functioning in the elderly. Am J Epidemiol 124, 372-388.

31. Zigmond AS \& Snaith RP (1983) The hospital anxiety and depression scale. Acta Psychiatr Scand 67, 361-370.

32. Bunevicius A, Peceliuniene J, Mickuviene N, et al. (2007) Screening for depression and anxiety disorders in primary care patients. Depress Anxiety 24, 455-460.

33. Kriegsman DM, Penninx BW, van Eijk JT, et al. (1996) Selfreports and general practitioner information on the presence of chronic diseases in community dwelling elderly. A study on the accuracy of patients' self-reports and on determinants of inaccuracy. J Clin Epidemiol 49, 1407-1417.

34. Hunt SM, McEwen J \& McKenna SP (1985) Measuring health status: a new tool for clinicians and epidemiologists. $J R$ Coll Gen Pract 35, 185-188.

35. Guralnik JM, Simonsick EM, Ferrucci L, et al. (1994) A short physical performance battery assessing lower extremity function: association with self-reported disability and prediction of mortality and nursing home admission. J Gerontol 49 , M85-M94

36. Verghese J, Wang C, Xue X, et al. (2008) Self-reported difficulty in climbing up or down stairs in nondisabled elderly. Arch Phys Med Rehabil 89, 100-104.

37. Ostbye T, Taylor DH \& Jung SH (2002) A longitudinal study of the effects of tobacco smoking and other modifiable risk factors on ill health in middle-aged and old Americans: results from the Health and Retirement Study and Asset and Health Dynamics among the Oldest Old survey. Prev Med 34, 334-345.

38. Garretsen HFL \& Knibbe RA (1983) Alcohol Prevalentie Onderzoek Rotterdam/Limburg, landelijk eindrapport (Alcohol Prevalence Study Rotterdam/Limburg, national report). Leidschendam: Ministerie van Welzijn, Volksgezondheid en Cultuur.

39. Stel VS, Smit JH, Pluijm SM, et al. (2004) Comparison of the LASA Physical Activity Questionnaire with a 7-day diary and pedometer. J Clin Epidemiol 57, 252-258.

40. de Jong-Gierveld J \& Kamphuis F (1985) The development of a Rasch-type loneliness scale. Appl Psych Meas 9, 289-299.

41. Comijs HC, Dik MG, Aartsen MJ, et al. (2005) The impact of change in cognitive functioning and cognitive decline on disability, well-being, and the use of healthcare services in 
older persons. Results of Longitudinal Aging Study Amsterdam. Dement Geriatr Cogn Disord 19, 316-323.

42. Chatterjee S, Hadi AS \& Price B (2000) Regression Analysis by Example, 3rd ed. New York: John Wiley and Sons.

43. Hickson M (2006) Malnutrition and ageing. Postgrad Med J 82, 2-8.

44. Chen LY \& Hardy CL (2009) Alcohol consumption and health status in older adults: a longitudinal analysis. J Aging Health 21, 824-847.

45. Sadakane A, Gotoh T, Ishikawa S, et al. (2009) Amount and frequency of alcohol consumption and all-cause mortality in a Japanese population: the JMS Cohort Study. J Epidemiol 19, $107-115$.

46. Chen CC, Schilling LS \& Lyder CH (2001) A concept analysis of malnutrition in the elderly. $J$ Adv Nurs 36, 131-142.
47. Sergi G, Perissinotto E, Pisent C, et al. (2005) An adequate threshold for body mass index to detect underweight condition in elderly persons: the Italian Longitudinal Study on Aging (ILSA). J Gerontol A Biol Sci Med Sci 60, 866-871.

48. Stratton RJ, Green CJ \& Elia M (2003) Disease-related Malnutrition: An Evidence-Based Approach to Treatment. Wallingford, UK: CABI Publishing.

49. Wannamethee SG, Shaper AG, Whincup PH, et al. (2000) Characteristics of older men who lose weight intentionally or unintentionally. Am J Epidemiol 151, 667-675.

50. McCormack P (1997) Undernutrition in the elderly population living at home in the community: a review of the literature. J Adv Nurs 26, 856-863.

51. Kubrak C \& Jensen L (2007) Malnutrition in acute care patients: a narrative review. Int J Nurs Stud 44, 1036-1054. 